\title{
Discrepancies between radiological and histological findings in preoperative core needle (CNB) and vacuum-assisted (VAB) breast biopsies
}

\author{
Inna Jörg ${ }^{1,5} \cdot$ Jann Wieler $^{2} \cdot$ Constanze Elfgen ${ }^{3,4} \cdot$ Kristina Bolten $^{5} \cdot$ Claudia Hutzli $^{6} \cdot$ Julia Talimi $^{7,8}$. \\ Denise Vorburger ${ }^{7,8} \cdot$ Matthias Choschzick $^{9} \cdot$ Linda Moskovszky $^{9} \cdot$ Konstantin Dedes $^{7,8} \cdot$ Zsuzsanna Varga $^{8,9}$ (i)
}

Received: 27 July 2020 / Accepted: 22 November 2020 / Published online: 7 December 2020

(c) The Author(s) 2020

\begin{abstract}
Background Ultrasound (US)-guided breast biopsy is a routine diagnostic method used to correlate imaging finding to a histological diagnosis which is still the gold standard in preoperative diagnostics. The accuracy of US-guided breast biopsies relies on a precise radiologic-histopathologic correlation, which is discussed amongst an interdisciplinary team of gynecologists, radiologists and pathologists. However, false-negative or non-diagnostic biopsy results occur. Hence, a thorough and honest discussion to clarify the reason for discrepancies and to decide the next diagnostic step between specialists of the different disciplines is warranted. In this retrospective study, we analyzed discrepant findings between imaging and pathology results on preoperative breast biopsies.

Methods Core and vacuum-assisted breast biopsies from 232 patients were included in this study. Inclusion criteria were (1) non-diagnostic (B1) category on histology independent from imaging category and (2) histological benign (B2) category with a BIRADS 5 (Breast Imaging Reporting and Data System) rating on imaging. Histological diagnoses were retrieved from all cases. Follow-up data were available in most cases.

Results 138 biopsies were classified as B1, 94 biopsies as B2 category. 51 of 138 B1 cases (37\%) underwent re-biopsy. Rebiopsy found malignancy (B5) in 19 of 51 cases, and B3/4 (premalignant) lesions in 3 of 51 cases. All B2 cases underwent second-look imaging-diagnosis, in 57 of 94 cases (66\%) consecutive direct surgery or re-biopsy. Of these, malignancy was diagnosed histologically in 26 of 57 cases $(45.6 \%)$.

Conclusion Determining imaging-pathology concordance after US-guided breast biopsy is essential. Discrepant cases and further diagnostic steps need to be discussed with an interdisciplinary approach.
\end{abstract}

Keywords Breast biopsy · Discrepancy · Imaging · Histology

Zsuzsanna Varga

zsuzsanna.varga@usz.ch

1 Department of Gynecology, Hospital Triemli, Zurich, Switzerland

2 Institute of Diagnostic Radiology, University Hospital Zurich, Zurich, Switzerland

3 Breast Center Seefeld, Zurich, Switzerland

4 Department of Radiology, University Witten-Herdecke, Witten, Germany

5 Department of Gynecology, Hospital Zollikerberg, Zollikerberg, Switzerland
6 Department of Gynecology, Hospital Männedorf, Zurich, Switzerland

7 Breast Center, University Hospital Zurich, Zurich, Switzerland

8 Comprehensive Cancer Center Zurich, University Hospital Zurich, Zurich, Switzerland

$9 \quad$ Institute of Pathology and Molecular Pathology, University Hospital Zurich, Schmelzbergstrasse 12, 8091 Zurich, Switzerland 


\section{Introduction}

US-guided biopsy on palpable and non-palpable breast lesions is a routine diagnostic tool in the preoperative setting. Core needle biopsy $(\mathrm{CNB})$ is less invasive than vacuum-assisted biopsy (VAB) and therefore fewer surgical complications are expected (Mihalik et al. 2010). In the case of benign findings in the biopsy, unnecessary surgery can be avoided. In addition, the cost of a biopsy is lower than that of an open surgical excision (Kim et al. 2008). In case of malignant findings, weekly preoperative senology boards provide recommendations on the basis of which individual options can then be discussed with a patient. These also depend on age, tumor stage, receptor status and of course, the patient's wishes.

The key of a successful core biopsy workflow is an excellent communication and a high level of agreement among attending radiologists, pathologists and gynecologists (Hahn et al. 2012; Parikh and Tickmann 2005). The breast sonographer is the most experienced in evaluation of abnormal findings, thus playing an essential role in the diagnostic process. Preferably, the same person who detected the lesion concurrently performs the biopsy to ensure accurate analysis of the lesion. This continuity helps to ensure the lesion recommended for biopsy is the one that actually undergoes biopsy as well as a direct assessment of its technical adequacy. The pathologist is crucial in assessing and communicating the quantitative and qualitative aspects of the biopsy. Literature shows the frequency of missed cancers in trials ranges from 0.3 to $8.2 \%$, with approximately $70 \%$ being identified immediately after core biopsy. Thirty percent are delayed falsenegative stereotactic breast biopsies where a further biopsy is needed in 9-18\% of patients (Mihalik et al. 2010).

Since the 2003 edition of the BIRADS was published, the BIRADS lexicon and classification have proven to be useful in predicting the likelihood of malignancy in radiologically assessed breast lesions (Kim et al. 2008; Lee et al. 2008; Park et al. 2018). Each BIRADS assessment category indicates an anticipated likelihood of malignancy, which is based on a thorough evaluation of the imaging features Kim et al. 2008, Lee et al. 2008.

Category 3 (probably benign) is reserved for specific imaging findings known to have a likelihood of malignancy of $>0 \%$ but $\leq 2 \%$. Such lesions include solid masses with a circumscribed margin, oval shape and parallel orientation; complicated cysts and clustered microcysts (Mendelson et al. 2013). Although the recommended management is imaging follow-up, CNB can be performed under certain circumstances. For category 3 lesions, a benign (B2) biopsy result can be regarded as concordant with the images. Malignant biopsy results (B5) are considered to be discordant, but the sonographic features should be reviewed for subtle suspicious imaging features that might have been overlooked in the first place (Youk et al. 2011).

Category 4 (suspicious of malignancy) covers a wide range of likelihood of malignancy, ranging from 2 to $95 \%$. Thus, almost all recommendations for image-guided breast interventions come from assessments made using this category. Starting in the fourth edition of BIRADS and maintained in its recent fifth edition, category 4 is subdivided into 4A, 4B, and 4C (Mendelson et al. 2013). The range of the likelihood of malignancy is $>2 \%$ and $\leq 10 \%$ for category $4 \mathrm{~A},>10 \%$ and $\leq 50 \%$ for category $4 \mathrm{~B}$, and $>50 \%$ and $<95 \%$ for category $4 \mathrm{C}$. Although the subcategorization of sonographic BIRADS category 4 has been reported to be useful in predicting the likelihood of malignancy, established objective criteria do not exist for the subcategories and interobserver agreement has been shown to be poor (Mihalik et al. 2010; Youk et al. 2010).

Category 5 (highly suggestive of malignancy) carries a very high probability ( $\geq 95 \%)$ of malignancy, and any benign percutaneous tissue diagnosis should be considered discordant.

The histopathological B classification is defined in an analogous way, such as B1 (not diagnostic), B2 (benign), B3 (lesions with uncertain potential), B4 (suspicious of malignancy) and B5 (malignant) (AGO (2020); S3 Leitlinien 2020; Breast Cancer Screening Program NSH 2020).

In this study, we systematically analyzed discrepant diagnoses between radiological and histological findings, focusing on non-diagnostic biopsies (in all BIRADS categories) and on malignant imaging diagnosis (BIRADS 5) with histologically benign lesions (B2 lesions).

\section{Materials and methods}

\section{Patient cohort}

In total, 232 patients were included in this study. The cohort was retrieved from diagnostic files from the Institute of Pathology and Molecular Pathology, University Hospital Zurich, where between 2010 and 2019 more than 10,000 diagnostic breast biopsies were performed.

The study was covered by a valid ethical approval (Zurich Cantonal Ethical Committee, KEK-2012-554 including regulation on informed consents).

Following groups were analyzed:

First group: Patients who were biopsied because of radiological lesions classified from BIRADS 2 to BIRADS 5, where a B1 lesion was reported in the histology. 
Second group: Patients who were biopsied because of radiological lesions classified as BIRADS 5 where a B2 lesion was diagnosed in the histology.

\section{Results}

\section{B1 histological findings and any BIRADS category}

This group encompassed 138 cases. Patients underwent breast core biopsies because of radiological findings from BIRADS 2 to BIRADS 5 lesion and histological results that showed non-diagnostic B1 lesions.

A

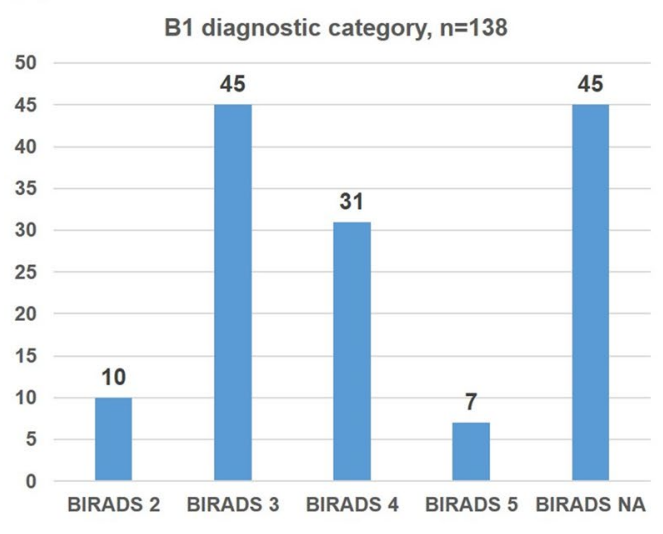

Ten biopsies were carried out for BIRADS 2 lesion, 45 biopsies for BIRADS 3, 31 biopsies for BIRADS 4 and 7 biopsies for BIRADS 5 imaging categories. In 45 patients, the radiological findings were suspicious for malignancy without documented BIRADS classification (Fig. 1a).

The BIRADS category prior to the US-guided CNB was established by ultrasound diagnostics (in 129 cases), ultrasound in addition to mammography in 7 cases, and ultrasound in addition to MRI in 2 cases. The initial biopsy was always US-guided CNB with 3-5 cores.

Histological findings showed fat/connective tissue in 60 cases (43\%) followed by skeletal muscles ( 5 cases), scar tissue ( 3 cases), normal breast tissue (11 cases), ductectasia ( 2 cases), fibrosis ( 35 cases), inflammation ( 1 case) and necrosis ( 1 case). In 23 cases, there was no evidence of breast tissue in the biopsy (Fig. 1b).

\section{B}

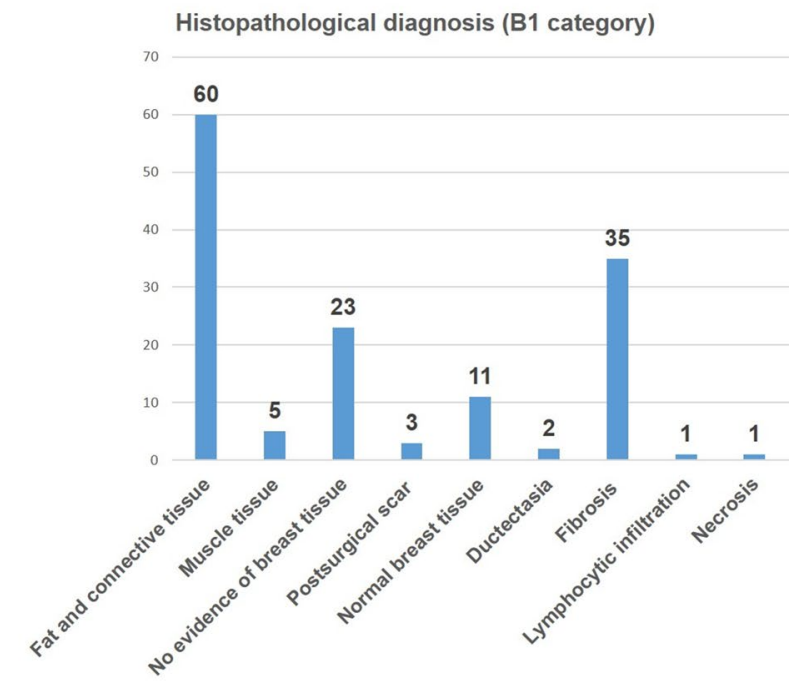

Fig. 1 The following diagnostic imaging (a) and histological (b) diagnoses were found in the primary biopsy with B1 diagnostic category

Fig. 2 Re-biopsy rate after B1 histological category (a) and distributions of B-category in re-biopsies after B1 diagnostic category (b)
A

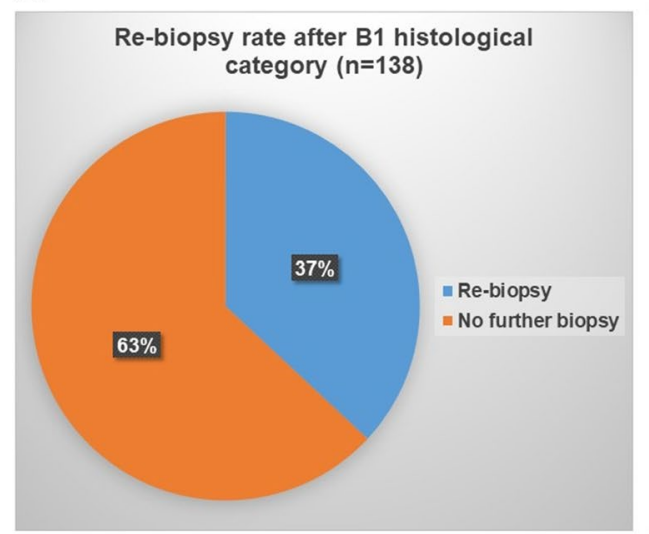

B

Re-biopsy histological categories after B1 diagnosis $(n=51)$

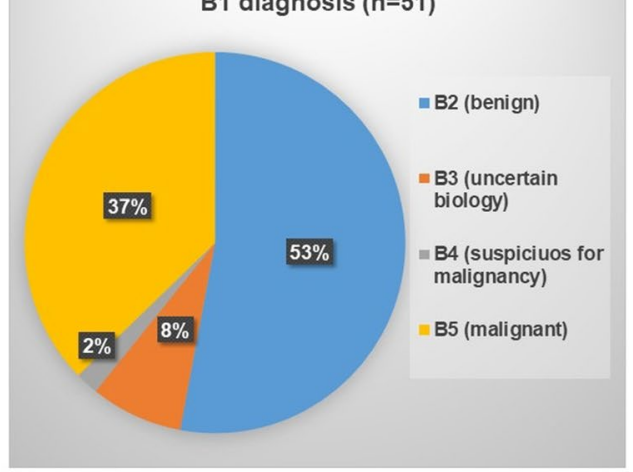


Table 1 Histological diagnoses in re-biopsies after B1 category

\begin{tabular}{lcl}
\hline $\begin{array}{l}\text { Re-biopsy category (after B1 } \\
\text { diagnosis) }\end{array}$ & $n$ & Histology \\
\hline B2 & $27(53 \%)$ & Benign histology \\
B3 & $4(8 \%)$ & Classical lobular neoplasia (LN) $(n=2)$ \\
& & Flat epithelial atypia (FEA) $(n=2)$ \\
B4 & $1(2 \%)$ & Atypical ductal hyperplasia (ADH) \\
B5 & $19(37 \%)$ & DCIS $(n=3)$ \\
& & Solid papillary carcinoma $(n=1)$ \\
& & NST carcinoma $(n=11)$ \\
& & Lobular carcinoma $(n=4)$ \\
\hline
\end{tabular}

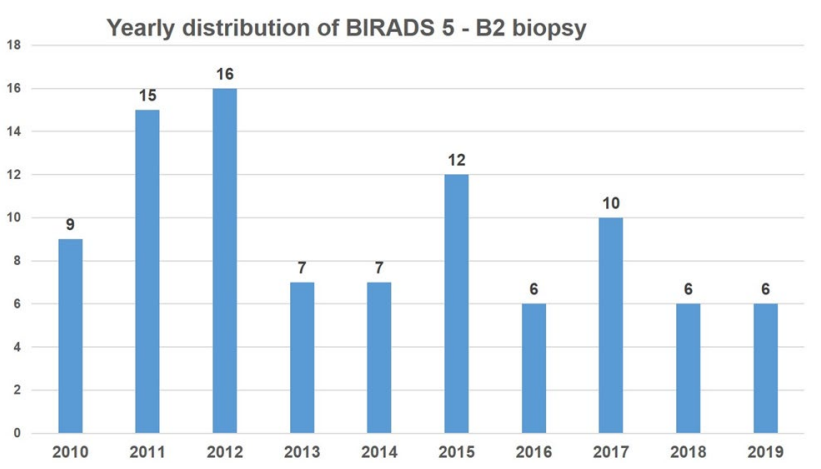

Fig. 3 Yearly distribution of BIRADS 5-B2 biopsy categories over a 10 -year period

Table 2 Histological diagnoses in the discrepant categories BIRADS 5 and B2 benign histology $(n=94)$

\begin{tabular}{lr}
\hline Scar/scarred fibrosis & 3 \\
Adenosis & 18 \\
Fibrosis & 39 \\
Chronic inflammation & 7 \\
Fibroadenoma & 10 \\
Fatty necrosis & 7 \\
Mastopathic changes, & 10 \\
$\quad$ usual ductal hyperplasia & \\
(UDH) & \\
\hline
\end{tabular}

51 out of 138 patients (37\%) underwent re-biopsy (Fig. 2a).

Out of the 51 cases which were re-biopsied, 19 (37\%) were diagnosed as B5, one (2\%) as B4, $4(8 \%)$ as B3 and $27(53 \%)$ as benign lesions (Table 1, Fig. 2b).

\section{Histological B2 category and radiological BIRADS 5 lesions}

This group consisted of patients with BIRADS 5 in imaging and a B2 diagnosis in histology. We identified 94 biopsies matching this category. The distribution of discrepancies between BIRADS 5 and histologically benign lesions varied from 6 to 16 cases per year (Fig. 3). In more than half of these cases (55\%), mastopathic changes were identified: fibrosis in 39 of 94 patients (36\%) and adenosis in 18 of 94 cases (19\%). Other frequent diagnoses included fibroadenoma, fat tissue necrosis, chronic inflammation and scar formation in the remaining $45 \%$ of B2 biopsies (Table 2). The BIRADS category prior to the US-guided CNB was established on ultrasound in 83 cases, ultrasound in addition to mammography in 9 cases, ultrasound in addition to MRI in 1 case and ultrasound in addition to PET CT in 1 further case. Initial biopsy was always US-guided CNB with 4-5 cores.

In 57 of the 94 cases, further diagnostics were carried out including a radiology-pathology correlation, US and in some cases MRI (60.6\% of all BIRADS 5 cases). Interestingly, from 2010 to 2012, radiologically suspicious but histologically benign (B2) lesions most frequently were handled by open surgery, while from 2012 to 2016, a re-biopsy with CNB was performed, and from 2017 to 2019 , VAB increasingly was used for discordant findings (Fig. 4a). Of the 57 cases, which were followed up by further diagnostics as described above, malignancy was confirmed histologically in 26 cases (45.6\%) (Fig. 4b).

\section{Discussion}

In this study, we analyzed cases in which a discordance between radiological (probably malignant or malignant) and histological (benign) findings was observed. A case was included when imaging features of a lesion that were suspicious for malignancy (i.e., BIRADS category 4 or 5 ), corresponded to benign histology (B2 category) in the CNB. The reported percentages of imaging-pathology discordant lesions among breast CNB range from 2.0 to $19.2 \%$ (Soyder et al. 2015; Sohn et al. 2014; Youk et al. 2010; Son et al. 2011). Histologically benign lesions with speculated findings, such as granular cell tumor, sclerosing adenosis, post-surgical scar, fatty necrosis, mastitis, diabetic mastopathy and sarcoidosis, can mimic malignancy on US (Cho and Park 2013; Kim et al. 2007). In our cohort, approximately half of BIRADS- 5 cases turned out to be malignant 
Fig. 4 Repeat diagnostic procedure after benign (B2) histology at BIRADS 5 imaging category (a) and malignancy rate in re-biopsies/primary surgery after benign (B2) histology at BIRADS 5 imaging category (b)
A

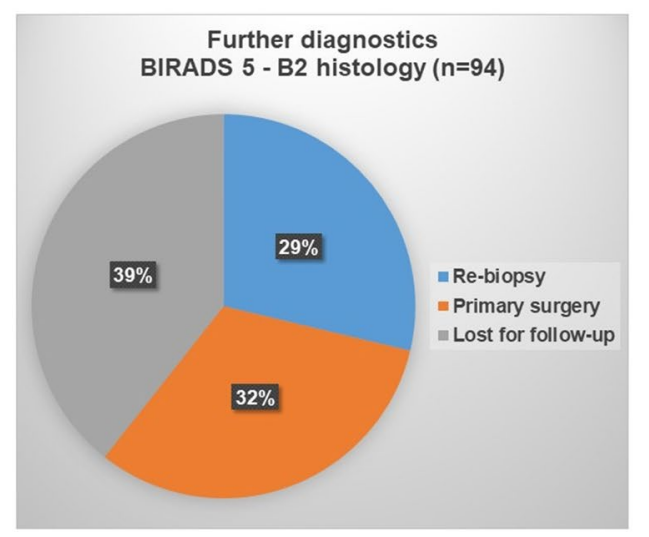

B

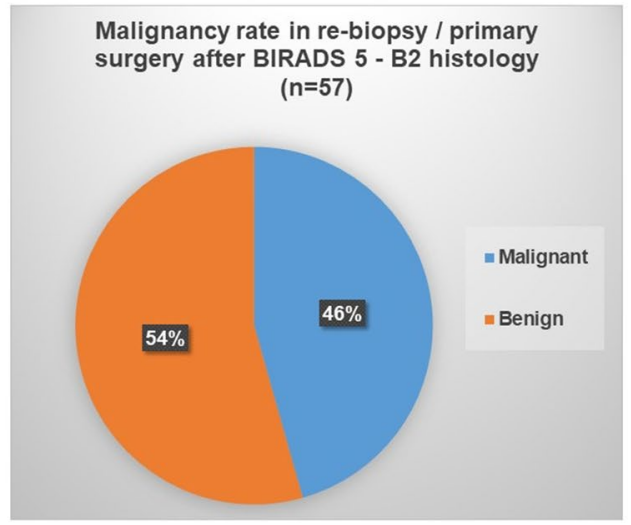

in re-biopsy or in open surgery, and the other half were confirmed benign.

The histopathological B classification is defined in several classification systems, such as the NHS Breast Cancer Screening program, the AGO guidelines, the $\mathrm{S} 3$ guidelines and also by the Minimal Invasive Breast Biopsy (MIBB) classification (AGO (2020); S3 Leitlinien 2020; Breast Cancer Screening Program NSH 2020). Category B1 is defined as normal tissue, lack of orientating breast parenchyma or insufficient tissue. Category B2 applies to benign lesions (such as fibroadenoma, usual ductal hyperplasia, fibrosis and cysts, among others). Category B3 encompasses biologically uncertain lesions (such as flat epithelial atypia, atypical ductal hyperplasia, classical lobular neoplasia, radial scar and benign/borderline phyllodes tumors). Category B4 is usually applied when a DCIS (or at least an ADH) is suspected, and category B5 describes malignancy (AGO 2020; S3 Leitlinien 2020).

The question of whether the BIRADS 5 category on ultrasound and/or mammography scans is correctly applied or is set too high for discrepant lesions, can only be answered by directly correlating the histological and the imaging findings in each given case (Parikh and Tickmann 2005; Son et al. 2011; Park et al. 2018).

Category B1 with normal breast tissue may indicate that the target lesion was not reached by the US-guided CNB. The documentation of the performance of the biopsy with regard to the US image (an ultrasound picture of the biopsy needle within the target lesion) is therefore of utmost importance. Sufficient experience in US-guided CNB has been shown to decrease the rate of misguided target lesions ( $\mathrm{Li}$ et al. 2010; Mihalik et al. 2010). Especially in discordant cases, the size of the CNB should be considered as thinner needles may potentially contribute to missed lesions ( $\mathrm{Li}$ et al. 2010; Mihalik et al. 2010).

Sonographic findings in special situations, such as scarring-as well as post-operative and post-irradiation connective tissue changes-may pose an even more difficult imaging interpretation, especially in view of the differential diagnosis of a recurrent lesion (Park et al. 2018). In discrepant cases, it is also essential for pathologists to re-view B1 and B2 diagnostic categories on the histological slides.

The interdisciplinary direct communication and feedback between the radiologist/gynecologist and pathologist is of utmost importance to enhance the learning effect and to clarify the reason for discrepant findings (Park et al. 2018; Mihalik et al. 2010; Soyder et al. 2015; Sohn et al. 2014; Youk et al. 2010).

In our study, more than half of initially discordant lesions that were biopsied with US-guided CNB were confirmed be malignant in subsequent open excision, VAB or re-biopsy (Mihalik et al. 2010; Soyder et al. 2015; Sohn et al. 2014; Youk et al. 2010). Discordant benign histological findings need to be immediately discussed between the performing radiologist/senologists and the interpreting pathologist, ideally even within an interdisciplinary preoperative senological board to clarify the discrepancy. Based on the decision of the multidisciplinary board, the radiologist should communicate with the referring physician as well as with the patient and discuss the need-or the lack thereof-for a repeated biopsy (Mihalik et al. 2010; Soyder et al. 2015; Sohn et al. 2014; Youk et al. 2010; Park et al. 2018; Parikh and Tickmann 2005).

In addition to CNB, a US-guided VAB has been shown to be a valuable alternative to open surgery for breast lesions with imaging-pathology discordance with an upgrade rate from 4.6 to $22.7 \%$ (Kim et al. 2012; Li et al. 2010; Wang et al. 2011). Therefore, both CNB and US-guided VAB can be an option for repeated biopsies of discordant benign lesions. The best biopsy method should be determined individually after consultation among the radiologists, pathologists, referring physicians and the patient. 


\section{Conclusion}

US-guided breast CNB is an accurate method for diagnosing breast cancer, with a false-negative rate ranging from 0.1 to $2.5 \%$. Determining imaging-pathology concordance after US-guided breast CNB is essential and needs to be addressed within an interdisciplinary preoperative conference. Discrepant findings should undergo a second-look review of imaging and histological findings, and the next consecutive diagnostic step should be decided within an interdisciplinary team. Appropriate management, including active communication between the pathologist, referring physician and patient, should be carried out accordingly.

Funding Open access funding provided by University of Zurich.

Data availability All data are available upon request without restriction.

\section{Compliance with ethical standards}

Conflict of interest None of the authors have conflict of interest to declare.

Open Access This article is licensed under a Creative Commons Attribution 4.0 International License, which permits use, sharing, adaptation, distribution and reproduction in any medium or format, as long as you give appropriate credit to the original author(s) and the source, provide a link to the Creative Commons licence, and indicate if changes were made. The images or other third party material in this article are included in the article's Creative Commons licence, unless indicated otherwise in a credit line to the material. If material is not included in the article's Creative Commons licence and your intended use is not permitted by statutory regulation or exceeds the permitted use, you will need to obtain permission directly from the copyright holder. To view a copy of this licence, visit http://creativecommons.org/licenses/by/4.0/.

\section{References}

AGO (2020) https://www.ago-online.de/fileadmin/downloads/leitlinien /mamma/2020

Breast Cancer Screening Program NSH (2020) https://www.nhs.uk/ conditions/breast-cancer-screening

Cho SH, Park SH (2013) Mimickers of breast malignancy on breast sonography. J Ultrasound Med 32:2029-2036

Hahn M et al (2012) Interdisciplinary consensus recommendations. Ultraschall Med 33:366-371

Jung HK, Moon HJ, Kim MJ, Kim EK (2014) Benign core biopsy of probably benign breast lesions $2 \mathrm{~cm}$ or larger: correlation with excisional biopsy and long-term follow-up. Ultrasonography 33(3):200-205

Kim MJ, Kim EK, Lee JY, Youk JH, Park BW, Kim SI et al (2007) Breast lesions with imaging-histologic discordance during USguided 14G automated core biopsy: can the directional vacuumassisted removal replace the surgical excision? Initial findings. EurRadiol 17:2376-2383
Kim EK, Ko KH, Oh KK, Kwak JY, You JK, Kim MJ et al (2008) Clinical application of the BI-RADS final assessment to breast sonography in conjunction with mammography. AJR Am J Roentgenol 190:1209-1215

Kim MJ, Kim D, Jung W, Koo JS (2012) Histological analysis of benign breast imaging reporting and data system categories $4 \mathrm{c}$ and 5 breast lesions in imaging study. Yonsei Med J 53:1203-1210

Lee HJ, Kim EK, Kim MJ, Youk JH, Lee JY, Kang DR et al (2008) Observer variability of Breast Imaging Reporting and Data System (BI-RADS) for breast ultrasound. Eur J Radiol 65:293-298

Li JL, Wang ZL, Su L, Liu XJ, Tang J (2010) Breast lesions with ultrasound imaging-histologic discordance at 16-gauge core needle biopsy: can re-biopsy with 10-gauge vacuum-assisted system get definitive diagnosis? Breast 19:446-449

Mendelson EB, Bohm-Velez M, Berg WA, Whitman GJ, Feldman MI, Madjar H et al (2013) ACR BI-RADS ultrasound. In: D’Orsi CJ, Sickles EA, Mendelson EB, Morris EA (eds) ACR BI-RADS Atlas, Breast Imaging Reporting and Data System, 5th edn. American College of Radiology, Reston, pp 1-173

Mihalik JE, Krupka L, Davenport R, Tucker L, Toevs C, Smith RS (2010) The rate of imaging-histologic discordance of benign breast disease: a multidisciplinary approach to the management of discordance at a large university-based hospital. Am J Surg 199:319-323

Parikh J, Tickman R (2005) Image-guided tissue sampling: where radiology meets pathology. Breast J 11(6):403-409

Park VY, Kim E-K, Moon HJ, Yoon JH, Kim MJ (2018) Evaluating imaging-pathology concordance and discordance after ultrasoundguided breast biopsy. Ultrasonography 37:107-120

S3 Leitlinien (2020) https://www.leitlinienprogramm-onkologie.de/leitl inien/mammakarzinom/

Sohn YM, Yoon JH, Kim EK, Moon HJ, Kim MJ (2014) Percutaneous ultrasound-guided vacuum-assisted removal versus surgery for breast lesions showing imaging-histology discordance after ultrasound-guided core-needle biopsy. Korean J Radiol 15:697-703

Son EJ, Kim EK, Youk JH, Kim MJ, Kwak JY, Choi SH (2011) Imaging- histologic discordance after sonographically guided percutaneous breast biopsy: a prospective observational study. Ultrasound Med Biol 37:1771-1778

Soyder A, Taskin F, Ozbas S (2015) Imaging-histological discordance after sonographically guided percutaneous breast core biopsy. Breast Care (Basel) 10:33-37

Wang ZL, Liu G, Li JL, Su L, Liu XJ, Wang W et al (2011) Breast lesions with imaging-histologic discordance during 16-gauge core needle biopsy system: would vacuum-assisted removal get significantly more definitive histologic diagnosis than vacuum-assisted biopsy? Breast J 17:456-461

Youk JH, Kim E-K, Kim MJ, Lee JY, Oh KK (2007) Missed breast cancers at US-guided core needle biopsy: how to reduce them. RadioGraphics 27:79-94

Youk JH, Kim EK, Kim MJ, Kwak JY, Son EJ (2010) Analysis of false- negative results after US-guided 14-gauge core needle breast biopsy. EurRadiol 20:782-789

Youk JH, Kim EK, Kim MJ, Ko KH, Kwak JY, Son EJ et al (2011) Concordant or discordant? Imaging-pathology correlation in a sonography- guided core needle biopsy of a breast lesion. Korean J Radiol 12:232-240

Publisher's Note Springer Nature remains neutral with regard to jurisdictional claims in published maps and institutional affiliations. 